Kong, Hong Kong, Hong Kong; ${ }^{5}$ Department of Epidemiology and Social Medicine, Xuanwu Hospital, Capital Medical University, Beijing, China; ${ }^{6}$ Department of Preventive Medicine, Yonsei University College of Medicine, Seoul, Republic of Korea; ${ }^{7}$ Department of Health Science, Shiga University of Medical Science, Otsu, Shiga, Japan; ${ }^{8}$ Life-Related Disease Prevention Center, Otsu, Shiga, Japan

Background Elevated blood pressure and excess weight are established major risk factors for cardiovascular disease (CVD). Previous studies have suggested that hypertension is a greater cardiovascular hazard among obese compared with lean individuals, but the epidemiological evidence is conflicting.

Methods and Results The interaction between systolic blood pressure $(\mathrm{SBP})$ and $\mathrm{BMI}$ on fatal or non-fatal coronary heart disease (CHD), ischaemic stroke and hemorrhagic stroke was examined using pooled data from the Asia Pacific Cohort Studies Collaboration. Participants of the study were 419448 men and women aged $>30$ years at baseline. BMI was categorised into five groups $\left(12.0-18.4,18.5-22.9,23.0-24.9,25.0-29.9\right.$ and $\left.30.0-60.0 \mathrm{~kg} / \mathrm{m}^{2}\right)$. Cox proportional hazard models, stratified by sex and study, were used to estimate HRs adjusting for age and smoking status, and the interaction between SBP and BMI was assessed by likelihood ratio test. During 2619241 person-years of follow-up, there were 10877 CVD events (59\% in Asia, 34\% women, 71\% fatal). For all forms of CVD except haemorrhagic stroke, there was evidence of an antagonistic interaction between SBP and BMI such that the risks of subsequent $\mathrm{CHD}(p=0.01)$, ischaemic stroke $(p=0.03)$ and CVD $(p=0.001)$ associated with increases in SBP were higher in normal-weight individuals compared with obese individuals.

Conclusion Increased SBP is an important determinant of subsequent cardiovascular risk irrespective of body size and, in relative terms, lean individuals were shown to have a poorer prognosis for $\mathrm{CHD}$ and ischaemic stroke.

\section{P2-310 GAMMA-GLUTAMYLTRANSFERASE AS A BIOMARKER FOR OXIDATIVE STRESS, METABOLIC SYNDROME, AND ALCOHOL CONSUMPTION AND ITS ASSOCIATION WITH CANCER INCIDENCE}

doi:10.1136/jech.2011.142976k.43

${ }^{1} \mathrm{H}$ Ulmer, ${ }^{1} \mathrm{~S}$ Strohmaier, ${ }^{1} \mathrm{~W}$ Borena, ${ }^{1} \mathrm{M}$ Edlinger, ${ }^{2} \mathrm{H}$ Concin, ${ }^{2} \mathrm{G}$ Diem, ${ }^{3} \mathrm{C}$ Kelleher, ${ }^{4} \mathrm{G}$ Nagel. ${ }^{1}$ Innsbruck Medical University, Department of Medical Statistics, Informatics and Health Economics, Innsbruck, Austria; ${ }^{2}$ Agency for Preventive- and Social Medicine, Bregenz, Austria; ${ }^{3}$ University College Dublin, School of Public Health, Physiotherapy \& Population Science, Dublin, Ireland; ${ }^{4} U \mathrm{Im}$ University, Institute of Epidemiology, Ulm, Germany

Background Alcohol consumption, metabolic factors and oxidative stress have consistently been linked to cancer development. Gamma-glutamyltransferase (GGT) is a biomarker for adverse alcohol consumption and oxidative stress. It is highly related to metabolic factors such as hyperglycaemia, dislipidaemia and obesity. We therefore hypothesise that GGT is associated with cancer incidence at different sites.

Methods First visit measurements in 94628 adult women and 80224 men screened for metabolic risk factors as part of the Vorarlberg Health Monitoring \& Promotion Programme (VHM\&PP). During a median follow-up of 13 years, a total of 5136 incident cancers were diagnosed in men and 4665 in women. Sexspecific Cox proportional hazards models, adjusted for age, bodymass index and smoking were performed to estimate HRs and $95 \%$ CI per quintiles of GGT.

Results In males, there were associations (highest vs lowest quintiles) of GGT with liver cancer ( $\mathrm{HR}=16.50,4.00-68.19)$, cancers of the lip, oral cavity, pharynx and larynx $(\mathrm{HR}=3.80,2.33-6.20)$, oesophageal cancer $(\mathrm{HR}=2.39,1.01-5.72)$, pancreatic cancer
$(\mathrm{HR}=2.13,1.01-4.56)$, lung cancer $(\mathrm{HR}=2.04,1.55-2.70)$, bladder cancer $(\mathrm{HR}=1.76,1.11-2.77)$, kidney cancer $(\mathrm{HR}=1.61,0.92-2.82$, $p$ for trend $=0.009)$ and colorectal cancer $(H R=1.36,1.01-1.83)$. In females, the association was most pronounced in cervical cancer $(\mathrm{HR}=3.77$, 1.94-7.32), followed by lung cancer $(\mathrm{HR}=1.63$, 1.02-2.60), endometrial cancer $(\mathrm{HR}=1.42,0.98-2.05, \mathrm{p}$ for trend $=0.013)$ and breast cancer $(\mathrm{HR}=1.19,1.02-1.39)$.

Conclusions GGT is a highly promising marker for risk stratification in cancer prevention.

\section{P2-311 CONSISTENCY BETWEEN THE MEASUREMENTS OF CHRONIC MORBIDITY IN A HEALTH INTERVIEW SURVEY AND A POPULATION CENSUS}

doi:10.1136/jech.2011.142976k.44

${ }^{1} \mathrm{~J}$ Van der Heyden, ${ }^{*}{ }^{2} \mathrm{D}$ De Bacquer, ${ }^{2} \mathrm{~K}$ Van Herck, ${ }^{1} \mathrm{H}$ Van Oyen, ${ }^{1} \mathrm{~J}$ Tafforeau ${ }^{1}$ Scientific Institute of Public Health, Brussels, Belgium; ${ }^{2}$ Department of Public Health, Ghent University, Ghent, Belgium

Introduction The mode of data collection may affect the outcome of a health indicator. This study aimed to examine the consistency between answers to an identical question on chronic morbidity obtained through a face-to-face interview in a health interview survey (HIS) and a self-administered questionnaire from a population census.

Methods During the last quarter of 2001, 2710 people aged 15 years and older participated both to a census and a HIS in Belgium. An individual linkage was performed between the two data sources by using the National Population Registry ID number. Consistency of answers to the question on chronic morbidity was assessed by the $\kappa$-statistic.

Results The prevalence of chronic morbidity was $29.4 \%$ (HIS) and $26.6 \%$ (census). Consistency was relatively poor, with a $\kappa$-statistic of 0.56 ( $95 \%$ CI 0.52 to 0.60 ). The $\kappa$-statistic did not differ by gender, but was substantially lower among persons aged 75 years and older (0.44; $95 \%$ CI 0.41 to 0.48$)$ than among younger individuals $(0.55$; $95 \%$ CI 0.51 to 0.59$)$. The $\kappa$-statistic was also lower for non-Belgians (0.43; $95 \%$ CI 0.40 to 0.47$)$ than for Belgians (0.57; 95\% CI 0.54 to 0.61). Consistency differed further among educational groups, although no real educational gradient was observed.

Conclusion There was no satisfactory correlation between selfreported chronic morbidity data in the HIS and the population census. The consistency also differed across population subgroups. The mode of data collection appears to impact the estimates. Estimates and sociodemographic determinants of self-reported chronic morbidity should be interpreted cautiously.

\section{P2-312 ASPIRIN USE IN CARDIOVASCULAR DISEASE PREVENTION: A POPULATION-BASED STUDY}

doi:10.1136/jech.2011.142976k.45

${ }^{1} \mathrm{C}$ Vianna, ${ }^{2} \mathrm{D}$ González, ${ }^{1} \mathrm{~A}$ Matijasevich. ${ }^{1}$ Universidade Federal de Pelotas, Pelotas, Rio Grande do Sul, Brazil; ${ }^{2}$ Universidade Federal de Santa Catarina, Florianópolis, Santa Catarina, Brazil

Objective To estimate the prevalence of aspirin use in primary and secondary prevention of cardiovascular disease.

Methods Population-based cross-sectional study was carried out in Pelotas, Southern Brazil, between Jan and May/2010, with people aged $\geq 20$ years old. This study had two outcomes: aspirin use in primary prevention (people $\geq 40$ years old, with at least two risk factors: Hypertension, Diabetes Mellitus and/or hyperlipidaemia) and aspirin use in secondary prevention (previous history of stroke and/or angina/myocardial infarction). The outcomes were analysed 
based in demographic (gender, skin colour, age and marital status), socioeconomic (schooling and socioeconomic position) and lifestyle variables (smoking, physical inactivity, obesity and self-perception of health status). $\chi^{2}$ Test was used for bivariate analyses and Poisson regression for multivariate analyses, with design effect adjustment.

Results Prevalence of aspirin use in primary prevention was $24.8 \%$ (95\% CI 21.0 to 28.9 ) and $34.3 \%$ (95\% CI 29.5 to 39.4 ) for secondary prevention. In primary prevention, aspirin use was higher in nonwhites and older people and among those with worst self-perceived health. For secondary prevention, the highest aspirin use was reported by the oldest, wealthiest and formerly smoking people.

Conclusion Aspirin use prevalence was low in both primary and secondary prevention of cardiovascular diseases. Especially in secondary prevention, where there is no doubt about the indication of aspirin, the use of medication in this population was half of that found in the literature. Further studies are necessary to identify the causes for this low aspirin use for prevention of cardiovascular diseases.

\section{P2-313 RELATIVE MORTALITY FROM COMMON CANCERS AMONG PEOPLE WITH TYPE 2 DIABETES AND THE EFFECT OF SOCIO-ECONOMIC STATUS}

doi:10.1136/jech.2011.142976k.46

J Walker, S Wild.* University of Edinburgh, Edinburgh, UK

Introduction Socio-economic status (SES) is associated with both type 2 diabetes and cancer mortality and may confound their association.

Methods We performed a retrospective cohort study using a population-based national diabetes database for 35-84 year olds in Scotland for 2001-2007 and an area-based measure of SES. RRs for mortality from lung, colorectal, breast and prostate cancer among people with type 2 diabetes compared to the population without diabetes were estimated using Poisson regression.

Results Complete data were available for 210994 eligible people (99.4\%). There were 2081 deaths from lung cancer, 945 from colorectal cancer, 528 from breast cancer and 419 from prostate cancer. Age adjusted RR (95\% CI) for lung, colorectal and prostate or breast cancer were 0.93 (0.82 to 1.04), 1.35 (1.23 to 1.48), 0.97 (0.89 to 1.07 ) for men and 1.03 (0.91 to 1.17$), 1.34$ (1.20 to 1.50$), 1.49$ (1.35 to 1.65$)$ for women. Age and SES adjusted RR (95\% CI) for lung, colorectal and prostate or breast cancer were 0.90 (0.84 to $0.96), 1.33$ (1.22 to 1.47$), 0.97$ (0.88 to 1.07 ) for men and 0.98 (0.90 to 1.05 ), 1.34 (1.19 to 1.49 ), 1.49 (1.35 to 1.65 ) for women.

Conclusions Type 2 diabetes was associated with higher mortality from colorectal cancer and breast cancer. Adjusting for SES had a small effect on RR for lung cancer but almost no effect for other cancers suggesting that SES is not an important confounder of the association between type 2 diabetes and mortality from common cancers.

\section{P2-314 RELATIVE BODY FAT AND BLOOD PRESSURE IN CHILDREN: A POPULATION BASED INDIVIDUALLY MATCHED STUDY IN CHINA}

doi:10.1136/jech.2011.142976k.47

'Z Wang,* 2J Ma. 'School of Medicine, University of Queensland, Brisbane, Queensland, Australia; ${ }^{2}$ Institute of Child and Adolescent Health, Peking University Health Sciences, Beijing, China

Introduction Normative blood pressure (BP) percentiles in children recommended by the current guidelines vary with body size. Sepa- rating the effect of fat mass from that of total body size on BP is important but challenging because fat mass and body size are strongly correlated. The objective of this study is to assess the relationship of relative body fat and BP in children.

Methods Chinese children who had a high skinfold thickness were individually matched by age, sex, weight and height to an equal number of children with a low skinfold. Systolic and diastolic BP levels were compared between the high and low body fat groups.

Results 7066 pairs of children aged 7-18 years were obtained, including 3042 pairs of boys and 4024 pairs of girls. As a result of matching, the two groups had identical distributions of height, weight and body mass index. The difference between the high and low fat groups in systolic BP was small $(0.03,95 \%$ CI -0.29 to $0.36 \mathrm{~mm} \mathrm{Hg}$ ) and not statistically significant. Diastolic BP in the high body fat group was 0.68 (95\% CI 0.40 to 0.96$) \mathrm{mm} \mathrm{Hg}$ higher than that in the low body fat group.

Conclusion For a given body size as measured by height and weight, relative body fat has little impact on systolic BP levels in children. Systolic BP is driven by overall body size. The same amount of fat mass and lean mass may have a similar impact on blood pressure in children.

\section{P2-315 THE EFFECTIVENESS OF SYSTEMATIC SYMPTOM ASSESSMENT TO IMPROVE PATIENT CARE IN ONCOLOGY MEDICINE: A SYSTEMATIC REVIEW}

doi:10.1136/jech.2011.142976k.48

${ }^{1,2} \mathrm{X}$ Wang, ${ }^{*}{ }^{1,2} \mathrm{R}$ Viola. ${ }^{1}$ Queen's University, Kingston, Ontario, Canada; ${ }^{2}$ Kingston General Hospital, Kingston, Ontario, Canada; ${ }^{3}$ Hotel Dieu Hospital, Kingston, Ontario, Canada; ${ }^{4}$ Oueen's Cancer Research Institute, Kingston, Ontario, Canada

Background Cancer patients do not voluntarily reveal all symptoms they experience. By asking them about symptoms using a structured systematic method, clinicians can find out about other symptoms. However, does systematic symptom assessment result in improved symptom control?

Objectives To determine and identify the potential association between systematic symptom assessment and a cancer patient's well-being and to gather information for future research.

Methods Electronic bibliographic databases were searched to July 2009. Search sources included MEDLINE, EMBASE, HealthSTAR, CINAHL, Cochrane Database of Systematic Reviews, Health and Psychosocial Instruments and Proquest Dissertations and Theses. Criteria for inclusion were: published full papers in English reporting controlled clinical trials or systematic reviews examining the effects of systematic symptom assessment on cancer patients' well-being.

Results 54 articles required full paper review by two reviewers, from which another 13 papers were reviewed arising from the reference lists. Five studies conducted from 1991 to 2006 met the eligibility criteria and were included in the analysis. The studies found that in the implementation of systematic symptom assessment in oncology care provided improved care in terms of pain management, overall symptom distress, quality of life, and aspects of patient and physician communication.

Conclusions Given limitations presented in the five studies, it is impossible to draw firm conclusions. However, this review is able to conclude that systematic symptom assessment provides valuable information in the overall assessment of the patient and its feedback to the clinicians do project an overall improvement in the patient's physical and mental well-being. 